\title{
La CIENCIA POLÍtica EN ECUADOR: UN REFLEJO DE SU FRAGILIDAD DEM OCRÁTICA (1978-2005)
}

\author{
Andrés Mejía Acosta \\ University of British Columbia, Canada \\ Flavia Freidenberg \\ Universidad de Salamanca, España
}

Simón Pachano

Facultad Latinoamericana de Ciencias Sociales, Ecuador

\begin{abstract}
Resumen
Desde su transición a la democracia, Ecuador ha experimentado tres rupturas presidenciales, múltiples reformas institucionales, incluyendo una Asamblea Nacional Constituyente, y la aparición de un movimiento político indígena. Frente a la magnitud de estos eventos, cabe decir que el análisis político se ha construido en buena medida desde las calles, en las asambleas populares, 0 en los editoriales de los periódicos. Carente de una metodología en común, un fundamento empírico o una perspectiva comparada, la ciencia política en Ecuador no ha desarrollado un espacio de debate propio y se ha limitado a dar explicaciones post mortem de los fenómenos políticos. Este ensayo revisa la evolución temática del análisis político, analiza los factores institucionales que impiden la enseñanza y la investigación, e identifica líneas para fortalecer el desarrollo de la disciplina en el país.
\end{abstract}

\begin{abstract}
Since its democratic transition, Ecuador has experienced three presidential breakdowns, several institutional reforms including a National Assembly, and the surge of an indigenous political movement. Given the unparalleled sequence of political events in this country, it is fair to say that most of the political analysis has taken place on the streets, popular assemblies, or newspaper articles. Lacking a common methodology, rigorous empirical research, and a comparative perspective, political science in Ecuador - with notable exceptions- has been limited to providing post mortem explanations of major political events. This paper reviews the evolution of the themes, analyzes the institutional factors that hindered the teaching and research, and outlines the main factors to promote the development of political science in Ecuador.
\end{abstract}

PALABRAS CLAVE • Ciencia Política • Ecuador • Democracia • Análisis Político

\section{INTRODUCCIÓN}

La ciencia política ecuatoriana se encuentra en un largo e inconcluso proceso de gestación iniciado en la década de 1990. Los estudios de carácter político de los últimos 25 años han estado marcados por el convulsionado desarrollo de la política y por el predominio de enfoques provenientes de la sociología, la antropología y la filosofía. Desde el fin del gobierno militar en 1978, la democracia en Ecuador ha sido testigo de varias crisis presidenciales, tres de las cuales termina- 
ron con la salida abrupta del presidente, una Asamblea Nacional Constituyente, múltiples reformas electorales y legislativas, alta volatilidad de los gabinetes y un proceso de organización y movilización del movimiento indígena que culminó en la formación de un partido de origen étnico.

En este convulsionado ambiente, bien se puede decir que la política ecuatoriana se ha escrito en las calles, en las asambleas populares, en los editoriales de los periódicos, con una fuerza y una intensidad que han relegado a segundo plano la reflexión académica y han obstaculizado el desarrollo de la ciencia política, con su componente de análisis empírico y comparación sistemática. Por el contrario, la producción de esta disciplina en Ecuador ha tenido en su mayor parte un carácter reactivo, con un enfoque cortoplacista, generalmente narrativo, descriptivo y especulativo. Esta tendencia se ha visto reforzada por la ausencia de programas consolidados de ciencia política a nivel de pregrado y su debilidad a nivel de postgrado, así como por la falta de profesores con entrenamiento (y formación) en este campo y la carencia de un mecanismo de arbitraje que asegure la calidad de las publicaciones académicas (peer review). Esto ha llevado a que el entorno académico de la ciencia política sea limitado y débil, por no decir inexistente.

Actualmente, hay tendencias de reforma institucional que prometen adoptar un entorno favorable para fomentar la producción académica. Estos incluyen, en primer lugar, la reciente adopción de programas de ciencia política a nivel de postgrado en algunas instituciones. En segundo lugar, se nota un desarrollo de mejores y más detalladas bases de datos de indicadores sobre la realidad política ecuatoriana. En tercer lugar, hay mayor presencia e intercambio de académicos extranjeros que realizan trabajo de campo en el país y mayor número de estudiantes ecuatorianos en programas de postgrado en el exterior.

Para abordar este tema, el presente ensayo comienza con una revisión de los criterios usados para evaluar el desarrollo de la ciencia política en Ecuador. En la segunda parte se hace una repaso de la evolución de los estudios políticos en los últimos 25 años (con una breve referencia a las contribuciones anteriores), dentro de una periodización determinada por los avances académicos y con referencia a los temas predominantes en los análisis. La tercera parte explora los espacios y ámbitos de difusión de la disciplina. La cuarta analiza los aspectos estructurales relacionados con las universidades y los programas de estudio, así como el impacto de los estudios políticos en el medio. Y, finalmente, la última parte esboza una conclusión general sobre la cuestión tratada.

\section{EL MÉTODO DE LA CIENCIA POLÍTICA}

En Ecuador, la ciencia política se encuentra en un nivel de desarrollo incipiente. Cruzada por diferentes tipos de tensiones, que hacen pensar en la idea de "mesas separadas" - como lo señaló Almond para la academia estadounidense en la década de 1950-, la disciplina destaca por una serie de características que condicionan su institucionalización y su autonomía. En este contexto, se consideró conveniente utilizar cuatro criterios para evaluar el desarrollo del conocimiento científico, con aplicación especial al de la ciencia política ecuatoriana.

Salvo contadas excepciones, la producción de conocimiento político en Ecuador ha sido de naturaleza subjetiva y especulativa. Con ello se ha atentado contra la primera condición de validez de la investigación científica que establece que ésta sea objeto de verificación empírica, es decir, que 
las propuestas se asienten en la observación y la experiencia (Buttolph et al., 2001: 23). Pero, incluso cuando la investigación se ha hecho sobre evidencia empírica, generalmente no se comunican o explican los métodos usados ni se ofrecen las oportunidades para verificar o replicar los hallazgos. La ausencia de métodos y foros para el debate académico ha impedido la transmisión y la acumulación del conocimiento científico. Se puede afirmar, sin temor a equivocarse, que uno de los vacíos más grandes de la ciencia política en el país se encuentra en la ausencia de investigación empírica que permita la comprobación de los hallazgos y la validez de las hipótesis.

Una segunda característica de la investigación política en Ecuador es su alto contenido ideológico. De manera especial -pero no exclusivamente- las décadas de 1970 y de 1980 se caracterizaron por el sesgo prescriptivo o respondieron a objetivos situados fuera del mundo académico. Con ello, se ha impedido la implantación de otra de las reglas básicas de la producción científica, que consiste en la aspiración de construir conocimiento libre de prescripciones normativas 0 juicios de valor, y que se limite a ofrecer una explicación objetiva y factual del fenómeno observado. Si bien no se puede aspirar a producir conocimiento puro en un espacio vacío de valores, la actividad científica debe evitar un sesgo normativo o ideológico en la interpretación de los hallazgos empíricos.

Una tercera característica es la gran cantidad de investigaciones de carácter descriptivo, que no ofrecen explicaciones acerca de las razones que llevaron a que el objeto de análisis produjera 0 adoptara determinadas características. Sin explicaciones sistemáticas derivadas de un conjunto de hipótesis, y sin la correspondiente verificación empírica, la producción politológica se ha estacionado en el ámbito de lo descriptivo, y en el mejor de los casos, de lo inductivo. Con ello se reducen las posibilidades de alcanzar la condición de construir proposiciones generalizables que tengan además capacidad explicativa. El método científico descansa en su capacidad de elaborar inferencias causales a partir de la observación del fenómeno, para luego ofrecer una explicación que se derive lógicamente de un conjunto de proposiciones teóricas, que con la sola excepción del tema del populismo y, en menor medida, de la participación política de los indígenas, ha estado ausente.

Finalmente, la producción politológica padece de "parroquialismo", ya que el debate político gira en torno a sí mismo sin hacer referencia a la perspectiva comparada, sea ésta de tipo histórica 0 transversal. Una buena parte de las preguntas de investigación están determinadas por la coyuntura, o la "moda intelectual", sin considerar el entorno regional específico, la presencia de factores explicativos similares en países vecinos o procesos regionales más amplios. De esta manera, la proto ciencia política ecuatoriana ha evitado el método comparado y se ha condenado al estrecho provincialismo de una incipiente disciplina. Sin la existencia de un método propio de investigación, los estudios de procesos políticos en Ecuador se han diluido en las contribuciones de otras disciplinas en lugar de beneficiarse y enriquecerse de ellas. En un contexto donde la ciencia política es incipiente y débilmente institucionalizada, las contribuciones teóricas y metodológicas de la historia, la economía, la antropología y la sociología han reemplazado al (en lugar de coexistir con) análisis politológico. La ausencia de especificidad ha llevado, además, a trivializar los límites del objeto de estudio, al punto que se han aplicado de manera indiscriminada diferentes técnicas de análisis, marcos teóricos y enfoques científicos, todos agrupados bajo el nombre genérico de estudios políticos. 


\section{ENFOQUES Y EVOLUCIÓN TEMÁTICA DE LA CIENCIA POLÍTICA EN ECUADOR}

Para efectos del análisis, se distinguen tres períodos históricos: a) la etapa previa a la democracia; b) los estudios de sociología política de la década de 1980 hasta mediados de la década de 1990, y c) los primeros pasos de una ciencia política a partir de 1995.

\section{Enfoques estructurales (previos a la instauración democrática en 1978)}

Como se ha mencionado anteriormente, los antecedentes históricos en la materia fueron débiles y estuvieron por lo general marcados por el predominio de otras disciplinas y de las perspectivas omnicomprensivas de carácter estructural. El pensamiento político ecuatoriano de las décadas de 1960 y 1970 se orientó hacia las descripciones de los grandes procesos (Cueva, 1988; Hurtado, 1977; Egas, 1983; Maiguashca, 1994), intentando conseguir una interpretación de la especificidad nacional. Siguiendo la corriente predominante en América Latina de ese momento, aquellos primeros pasos se inscribieron en las visiones de conjunto, generalmente vinculadas a las explicaciones estructurales tanto de corte marxista como dependentista.

Solamente algunos trabajos aislados, reducidos por lo general a artículos en revistas de limitada circulación, se interesaron en fenómenos políticos tan importantes para el país como el populismo que, con la figura de Velasco Ibarra, había dominado el panorama nacional por cuatro décadas (Ojeda, 1971, 1983). La mayor parte de la producción en ciencias sociales, mientras tanto, se ocupaba del estudio de los temas agrarios y de las interpretaciones históricas.

En cuanto a los trabajos producidos des de el exterior, habría que señalar la contribución de George Blankstein (1951) como la primera obra que estudia a las instituciones políticas ecuatorianas desde una perspectiva comparada. Asimismo, los estudios seminales de John D. Martz sobre cultura política (1972) y de Samuel Fitch sobre el papel de los militares en los golpes de Estado (1977) fueron centrales entre los trabajos des de el exterior, toda vez que infirieron y propusieron conclusiones teóricas generales a partir del análisis del caso ecuatoriano.

\section{La etapa democrática}

Es innegable que el desarrollo de la ciencia política en un país está condicionado por la vigencia del sistema democrático (Alcántara, 1994; Almond, 2001). Sin democracia es prácticamente imposible que se produzca ciencia política. Ecuador es un buen ejemplo de ello, aunque también es una muestra de que la vigencia de un sistema democrático es una condición necesaria pero no suficiente para el desarrollo de la disciplina. Si bien en las décadas de 1980 y 1990 se ha dado un avance en la producción politológica en -y sobre- el país, también es cierto que la democracia no llevó en ese momento a la creación de programas de especialización que tuvieran como objetivo el desarrollo de la disciplina de manera autónoma ni tampoco a asociaciones que agruparan a quienes realizaban estudios de este tipo.

Durante la primera década y media de democracia se desarrollaron enfoques interesados en entender el proceso político que se iniciaba (Argones, 1986; Mills, 1984), sus actores (Icaza, 1983); sus instituciones (Acosta et al, 1984; Ayala, 1985; Pachano, 1991), y la cultura de la clase política (De la Torre, 1998; Burbano, 1998a). 
Sin duda, el estudio del populismo, en sus múltiples vertientes, ha tenido el mayor desarrollo conceptual y metodológico, convirtiéndose en uno de los temas más característicos de la ciencia política ecuatoriana. La producción sobre este tema ha adoptado estrategias analíticas diferenciadas y se ha enfocado sobre dimensiones específicas. Así, se encuentran estudios de populismo en cuanto a movimiento político (Cueva, 1988; Quintero, 1980; De la Torre, 2001); como discurso de interpelación a las masas (Burbano, 1989; Guerrero, 1990); como instrumento de liderazgo político (Martz, 1983; De la Torrre, 2000), como régimen de gobierno (Acosta, 1996), en relación al clientelismo (Menéndez Carrión, 1986; León, 1987; Guerrero, 1994; Pachano, 1999); respecto de las relaciones de dominación social y en su vertiente regional que lo vincula con la cultura política (Andrade, 2001). El contenido original y los enfoques innovadores desarrollados a partir de este tema han contribuido a comprender el fenómeno más allá del contexto ecuatoriano, lo cual ha mostrado por un momento la capacidad de la reflexión nacional para explicar fenómenos más generales.

Finalmente, la reflexión sobre la democracia constituyó el telón de fondo de los acercamientos al tema político en esta etapa (Bustamante, 1997; Isaacs, 1990; Menéndez Carrión, 1991; Pachano, 1996). Cabe destacar, sin embargo, que este análisis sobre las condiciones y el futuro de la democracia ha tenido serias limitaciones en la medida en que no se ha llegado a manejar un concepto compartido de democracia, ni siquiera en sus bases más generales. El sesgo ideológico de buena parte de los académicos ha sido un obstáculo en este sentido, ya que ha llevado al rechazo de perspectivas que son ampliamente aceptadas en la ciencia política contemporánea y que se asientan sobre procesos sostenidos de construcción conceptual y teórica.

\section{La etapa contemporánea}

La crisis política y social que culminó con el derrocamiento del Presidente Abdalá Bucaram dio inicio a una nueva etapa de los estudios políticos en Ecuador, concretamente a la gestación de una ciencia política que todavía no acaba de nacer. El cambio de enfoque se fundamentó en la noción de que hay procesos políticos que no dependen de macro-estructuras económicas o sociales, sino de aspectos más tangibles y concretos como los sistemas electorales, las reglas que conducen la vida de los partidos, el liderazgo político, el comportamiento electoral de los ciudadanos, las motivaciones individuales de los legisladores en el Congreso y la aparición de nuevos actores en el escenario político. Durante esta época, se resaltan tres vertientes de estudio, que no son las únicas pero sí las más desarrolladas desde la perspectiva de la ciencia política: instituciones políticas, cultura política, y la emergencia de nuevos actores políticos como los indígenas - analizada desde las dos perspectivas mencionadas.

Dentro del estudio de las instituciones políticas, resaltan los trabajos sobre elecciones, partidos y el sistema de partidos. Hay un notable interés en los procesos electorales (Quintero, 2002; Ardaya y Verdesoto, 1999; Pachano, 1998; León Velasco, 1992; Ibarra, 1994; Montúfar, 1990), el rendimiento electoral y la competencia de los partidos (Echeverría, 1994; Conaghan, 1995; Pachano, 1996, 2004; Mejía, 1998; Freidenberg y Alcántara, 2001; Freidenberg, 2003; Córdoba, 2003), la organización interna (Freidenberg, 2001), y su ideología (Bustamante, 1984; Freidenberg, 2005). También se han desarrollado algunos trabajos sobre los efectos del sistema electoral sobre el sistema de partidos (Pachano, 1999; Mejía, 1998; Sánchez, 1999), pero no lo suficientes como 
para poder identificar claramente los efectos que las reglas electorales tienen sobre las instituciones y la gobernabilidad.

Los enfoques institucionales se han adoptado para estudiar el funcionamiento del Congreso, el conflicto entre poderes y la formación de políticas públicas. En estos temas resaltan los análisis sobre los perfiles de los diputados, sus incentivos y carreras legislativas (Pachano, 1991; Mejía, 1999, 2003); la disciplina partidaria (Mejía, 2004); la relación entre el ejecutivo y legislativo y la formación de coaliciones legislativas (Burbano y Rowland, 1998; Mejía, 2000, 2004; SánchezParga, 1998; Landau, 2001), la gestión de gobierno y la política económica (Montúfar, 2000) y el papel de las instituciones sobre la formación de políticas públicas (Mejía et al., 2004). Esta línea de trabajo ha sido también recogida bajo el tema más amplio de la gobernabilidad (CORDES, 1996; 1999; Mejía, 2002; Echeverría, 1997).

El análisis de la cultura política ha sido abordado de manera rigurosa, a través de estudios de opinión pública. En esta línea se encuentra el Proyecto de Elites Parlamentarias en América Latina, dirigido por Manuel Alcántara Sáez desde la Universidad de Salamanca, que ha levantado encuestas sobre las actitudes de los diputados de los períodos 1996-1998, 1998-2002 y 2003-2006, para conocer sus percepciones sobre las instituciones (Mateos y Alcántara, 1997), su posición ideológica en los ejes de izquierda-derecha y el contenido programático de sus propuestas (Freidenberg, 2005). Asimismo, Selligson y Córdoba (2002) han desarrollado investigaciones a nivel de la ciudadanía, desde el Centro de Opinión Pública Latinoamericana de la Universidad de Pittsburgh. Existen además otros trabajos que han incorporado el uso de encuestas de opinión para desarrollar sus hipótesis como los de Baquero (1979); o Isaacs (1990).

El análisis de la cultura política desde el enfoque cualitativo se ha desarrollado para destacar la relevancia del factor regional y la generación de sub-culturas políticas regionales (Pachano, 1996; Bustamante, 2000), la identidad, diversidad étnica y la democracia (Guerrero, 1998); y la vinculación de la cultura política con la democracia a partir de la gobernabilidad (Burbano, 1998a; Bustamante, 1997), especialmente tras las crisis institucionales. Además, se distinguen estudios sobre los significados de las categorías sociales de uso común (Andrade, 2000) y el conjunto de representaciones simbólicas que ellas contienen.

Un tema que ha despertado creciente interés ha sido el surgimiento de los indígenas como actor social a inicios de la década de 1990 y el gran impacto que ha tenido para cuestionar y replantear la concepción y el funcionamiento de la democracia. En la última década, se han producido importantes análisis desde la perspectiva de la acción colectiva y las nuevas formas de representación política (Barrera, 2001; Massal y Bonilla, 2000), en cuanto a la activación de un partido político a partir de la movilización de un clivaje étnico tradicionalmente dormido en una sociedad en proceso de formación (Sánchez y Freidenberg, 1998; Freidenberg, 2004), sus implicaciones para la competencia electoral y partidaria (Mijeski y Beck, 1998), y otros temas que resaltan los dilemas de asimilación democrática planteados por el movimiento indígena (Van Cott, 1999; Yashar, 2005).

A pesar de su importancia en la escena política, el análisis de los grupos de interés y su influencia sobre las instituciones políticas ha recibido poca atención académica. Quizás una excepción en este sentido sea el trabajo pionero de Conaghan (1988) sobre la relaciones entre los industriales y el poder; el de las cámaras de la producción y la política de Naranjo (1993) o el de Corkil y Cubit (1992) sobre la relación 
entre capitalistas, tecnócratas y políticos. Tampoco se observan análisis desde la perspectiva de la acción racional, aunque dicho enfoque esté presente de manera tangencial en algunos análisis institucionales. También cabe citar, quizás como una contratendencia al análisis político sistemático, las múltiples contribuciones de coyuntura que han explicado desde una perspectiva periodística y narrativa los sucesos correspondientes a los plebiscitos (en 1994 y 1995), las reformas electorales y la Asamblea Nacional (en 1997 y 1998) y las rupturas presidenciales (en 1997, 2000 y 2005).

En general, los académicos ecuatorianos han continuado "sentándose en mesas separadas", con escasos intercambios entre ellos, con gran desconocimiento de la producción local y de los aportes externos (con excepción de débiles redes de difusión) y con escaso - por no decir nulodebate. Todo ello ha dificultado la construcción de una disciplina asentada sobre una comunidad académica estable, que pueda producir conocimiento acumulativo. Estas condiciones adversas han desembocado en prácticas alejadas de las rutinas científicas, sin congresos que permitieran debatir los avances dentro del propio campo y con una producción que se asiente sobre lo que se viene realizando a lo largo del tiempo. De esta manera, el mundo académico - que gira en torno a los estudios políticos- no ha podido construir los tres pilares básicos de una disciplina científica: orientación empírica, conocimiento acumulativo y comunidad académica.

\section{LA PRODUCCIÓN DE CIENCIA POLÍTICA: BIBLIOGRAFÍA EXISTENTE Y ESPACIOS DE DIFUSIÓN}

Una breve revisión de las publicaciones sobre temas políticos revela dos tendencias. La primera, la explosión y diversificación de investigaciones de carácter político a partir de 1995. La segunda, el relativo "parroquialismo" de los estudios sobre la realidad política ecuatoriana. Estos dos aspectos se desarrollan a continuación.

\section{La evolución y tipo de publicaciones en los últimos 25 años}

Para la elaboración de este texto se han construido bibliografías, se revisaron algunas previamente existentes y se exploraron los estudios presentados en conferencias regionales de ciencia política, instituciones académicas de Ecuador y programas de búsqueda bibliográfica. Esta revisión no pretende ofrecer una clasificación exhaustiva de la literatura existente pero constituye un primer intento de clasificar más de seiscientas publicaciones que hacen alguna referencia a procesos políticos en Ecuador 0 , si son estudios comparados, que usan el caso de Ecuador como base de referencia ${ }^{1}$.

Los datos verifican dos tendencias en este sentido. La primera, es una explosión de publicaciones a partir de la década de 1990, especialmente desde la segunda mitad. Entre 1991 y 2004, la producción de estudios fue de casi cuatro veces más (456) que los elaborados durante la década anterior (122). Más de la mitad de estudios de la muestra total $(55,6 \%)$ se publicaron a partir de la instalación -y posterior derrocamiento- del gobierno de Abdalá Bucaram en 1996. Este período coincide además con la aparición en escena del Movimiento indígena Pachakutik (1995) y el desarrollo de la Asamblea Nacional Constituyente (1998).

Para evitar una doble contabilidad en la recolección de datos, se incluyen libros editados con articulos de politica ecuatoriana como una sola contribución. Asimismo, se considera solamente la última versión publicada de un artículo en caso de que haya sido publicado en varios formatos. En total se contabilizaron 612 publicaciones. 
La segunda tendencia derivada del conteo de publicaciones es la diversificación de los formatos de estudio. En el período analizado se observa que más de la mitad del total de publicaciones contabilizadas son artículos de revistas (arbitradas y no arbitradas), y la cuarta parte fueron libros 0 monografías. El resto de referencias se divide entre capítulos en libros e informes o ensayos presentados en conferencias. Durante la década de 1980 hay una tendencia a producir casi exclusivamente artículos de revistas o monografías completas (libros). A partir de la década de 1990, hay una marcada tendencia hacia la diversificación de los formatos de publicación. Los formatos predominantes son desplazados por una mayor presencia de capítulos en libros editados - una buena parte en el exterior-, presentaciones en conferencias e informes para organismos 0 agencias de cooperación ${ }^{2}$. La Figura 1 ilustra la explosión y la diversidad de publicaciones.

FIGURA 1: Número y tipo de publicaciones en ciencia política o temas afines (1978-2004)

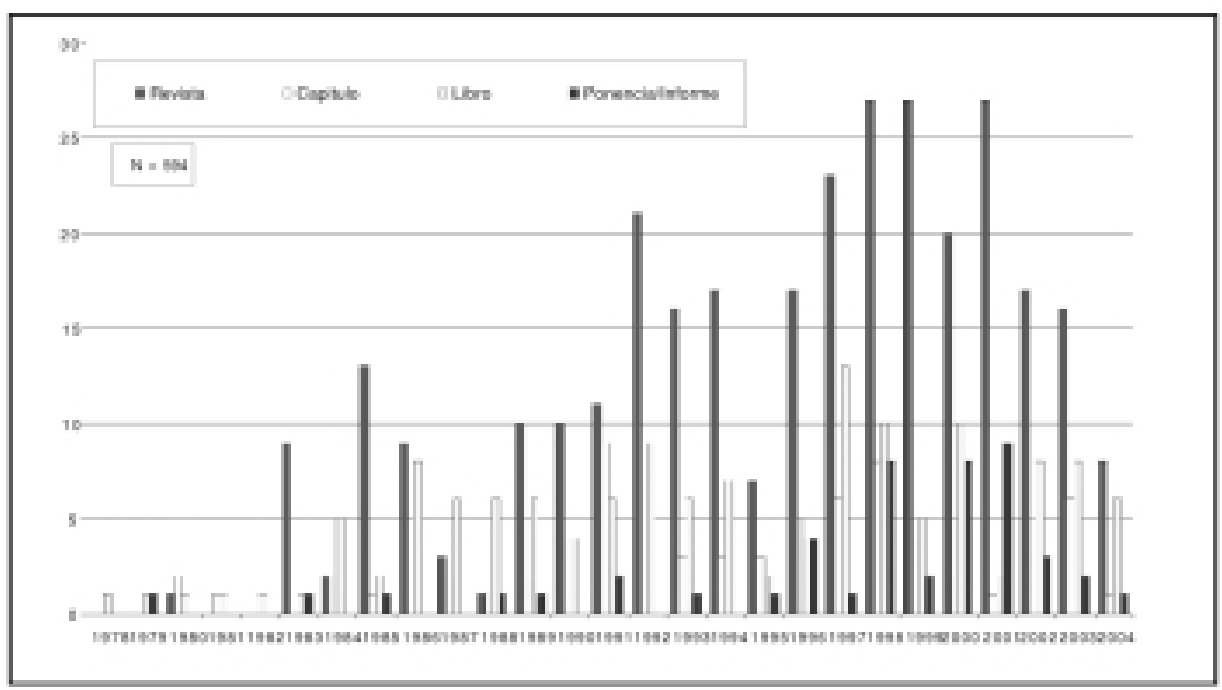

De las revistas donde se publican estudios políticos, las de uso más frecuente son Ecuador Debate, del Centro Andino de Acción Popular (CAAP), y la Revista Íconos de FLACSO. Ecuador Debate ha publicado casi la mitad de los artículos en revistas identificados en nuestra muestra, convirtiéndose en un puntal de la discusión de temas políticos, desde un enfoque ecléctico e interdisciplinario. Íconos es la primera en tener un sistema de arbitraje (peer review o doble ciego) y se halla en un proceso de profesionalización en los últimos años para cumplir con las exigencias internacionales de edición. Aún así, por el momento, ninguna revista se encuentra indexada en bases de datos internacionales (ISI).

\section{El espacio de publicaciones o el "parroquialismo" de la ciencia política}

Una de las causas por las que la ciencia política en Ecuador no ha florecido es que el debate académico -cuando existe- no trasciende las fronteras nacionales. Analizando los datos en función de su lugar de publicación, se encuentra que dos tercios de la producción total contabilizada

2 Un ejemplo es la serie de cuadernos de trabajo sobre gobernabilidad publicados por CORDES entre 1997 y 1999 , financiados por la Agencia de Cooperación Española. 
fueron impresos en Ecuador y, cerca del $80 \%$, si se incluye toda América Latina. Esta tendencia se refuerza por el hecho de que más del $80 \%$ de publicaciones aparecen en castellano, abandonando un espacio importante de difusión y debate de la problemática ecuatoriana en círculos anglosajones. La siguiente tabla ilustra estas tendencias.

TABLA 1: Número y porcentaje de publicaciones sobre Ecuador, por región e idioma

(1978-2004)

\begin{tabular}{lccccc}
\hline & \multicolumn{5}{c}{ Idioma } \\
\hline Región & Español & Inglés & Otro & \multicolumn{2}{c}{ Total } \\
\hline Ecuador & 396 & & & 396 & $66,4 \%$ \\
América Latina & 72 & & & 72 & $12,1 \%$ \\
Estados Unidos/Canadá & & 75 & & 75 & $12,6 \%$ \\
Europa & 30 & 17 & 6 & 53 & $8,9 \%$ \\
Total por Regiones & 498 & 92 & 6 & 596 & \\
\hline & $83,6 \%$ & $15,4 \%$ & $1,0 \%$ & $100,0 \%$ & $100,0 \%$ \\
\hline
\end{tabular}

La creciente presencia de ecuatorianistas en conferencias regionales como el Latin American Studies Association (LASA), y la creación de un capítulo de Ecuatorianistas dentro de esta Asociación, prometen revertir la entropía de los estudios y enriquecer la producción y los enfoques metodológicos de la ciencia política en Ecuador.

\section{EL CONTEXTO INSTITUCIONAL: LAS INSTITUCIONES DE EDUCACIÓN SUPERIOR Y LA CIENCIA POLÍTICA COMO PROFESIÓN}

Los estudiosos de procesos políticos ecuatorianos (dentro y fuera del país) tienen generalmente una doble frustración. Por un lado, el Ecuador no ha cesado de experimentar dramáticos procesos de quiebra democrática, constante inestabilidad y baja capacidad para procesar las demandas sociales y políticas, que han impedido rebasar las perspectivas de coyuntura y desarrollar análisis sistemáticos y comparados. Por otro, las instituciones académicas están en un proceso incipiente de consolidar mejores programas de ciencia política, pero aún no existe un espacio adecuado para desarrollar investigaciones rigurosas sobre la materia. Además, las instituciones académicas no han logrado establecer los incentivos profesionales de largo plazo para retener un creciente flujo de cientistas políticos que optan por desarrollar sus actividades profesionales en el sector privado, de gobierno 0 en el exterior.

\section{La enseñanza de la ciencia política}

Una de las causas de la debilidad de la ciencia política es la casi total ausencia de la carrera en los programas universitarios. Solamente tres universidades (San Francisco de Quito-USFQ, Casa Grande de Guayaquil y Cristiana Latinoamericana) tienen en su malla curricular una carrera de pregrado vinculada a algún aspecto de la especialidad. Sin embargo, en la USFQ está suspendida desde hace 
dos años y, en las otras dos, recién se han iniciado las actividades en el último año. Cinco universidades (Central y Católica de Quito, Estatal de Guayaquil, Vargas Torres de Esmeraldas y Técnica de Machala) ofrecen el título de Sociología y Ciencias Políticas, que en realidad corresponde a la primera disciplina. Solamente dos de ellas (Católica y Central de Quito) han tenido en algún momento, pero no de manera estable, la especialización en Ciencia Política como parte del programa de Sociología.

En el nivel de postgrado, la Universidad Católica del Ecuador ofreció dos promociones de la maestría en administración y ciencia política, dentro de un programa que ha sido descontinuado. La Facultad Latinoamericana de Ciencias Sociales, FLACSO-Ecuador, mantiene una maestría en ciencia política, que actualmente se encuentra en su tercera promoción. Anteriormente, esta maestría funcionó bajo el título de estudios políticos y de sociología política, lo que constituye una señal de la evolución hacia el campo de la ciencia política, con el correspondiente alejamiento de las visiones más generales e influidas por las otras disciplinas. La misma entidad realizó un curso de postgrado (en el nivel de diploma superior) con la Universidad de Cuenca. Así mismo, FLACSO inició en septiembre de 2004 un doctorado en ciencias sociales con especialización en ciencia política.

Existen pocos contactos entre las entidades de Educación Superior, pero éste no es un problema exclusivo del campo de la ciencia política. Paradójicamente, son más frecuentes los intercambios académicos con universidades norteamericanas (Estados Unidos y Canadá), europeas (especialmente de España, Francia y Alemania) y de otros países latinoamericanos que entre las instituciones ecuatorianas. Este es uno de los obstáculos para la conformación de una comunidad académica. Una de sus expresiones ha sido la ausencia de congresos y otros eventos que permitan el intercambio entre los profesionales de la disciplina, que ha comenzado a superarse débilmente con la celebración en los últimos años de los encuentros de la sección de Estudios Ecuatorianos de LASA en Quito.

\section{Los espacios profesionales}

Existen aproximadamente unos cincuenta ecuatorianos que estudian o han estudiado en el exterior en áreas afines a la ciencia política (gobierno, administración pública, estudios latinoamericanos). De ellos, aproximadamente la mitad ha vuelto a trabajar a Ecuador, la mayor parte como docentes en universidades como FLACSO, UASB, USFQ, Universidad Central, PUCE, Universidad del Azuay y Machala. El nivel de profesionalización de la ciencia política es bajo, toda vez que sus integrantes no comparten similares técnicas metodológicas ni tampoco una literatura común 3 . La baja profesionalización de la disciplina se debe en primer lugar, a la fuerte influencia de otras disciplinas (sociología política, antropología, derecho); a la desconexión entre lo que se produce en el exterior y lo que se produce en el país; a la ausencia de redes de politólogos que fomenten el desarrollo de la disciplina y a la carencia de una práctica científica que se asiente sobre el conocimiento acumulativo.

Un número creciente de los profesionales de la disciplina desarrolla actividades de asesoría y consultoría en instancias de toma de decisiones (gubernamentales, municipales, legislativas) y en organismos internacionales. Esto ha determinado que una parte considerable de los aportes y de las publicaciones respondan a objetivos propios de esos tipos de entidades y actividades, mas no a 
inquietudes estrictamente académicas. Por otra parte, muchos profesionales de la ciencia política han incursionado en los medios de comunicación, ya sea como columnistas estables o como analistas, y son requeridos con frecuencia para el análisis del momento político inmediato. Esto ha llevado a una vinculación muy estrecha con los hechos cotidianos, con la consecuente dificultad para tomar distancia y estructurar miradas de largo alcance, como lo demanda el trabajo científico.

A pesar de que han mejorado las condiciones laborales - lo que se expresa en que un grupo aún pequeño pero que tiende a incrementarse puede dedicarse a tiempo completo a las actividades académicas-, las posibilidades de inserción laboral siguen siendo un obstáculo para la profesionalización. Las remuneraciones relativamente bajas y la escasa valoración de la actividad académica (generalizada para todo el ámbito del quehacer científico) han configurado un campo profesional dominado por las actividades de consultoría y asesoría. De esta manera, los requerimientos del mercado laboral no son un incentivo para el desarrollo de la ciencia política en su concepción académica sino más bien para estudios políticos estrictamente limitados a objetivos específicos de carácter inmediato y de corto alcance. Entre otros efectos, esto lleva a conceder muy poco valor a la publicación en general, y en revistas especializadas en particular, de manera que hacerlo o no hacerlo finalmente se reduce a una decisión personal del académico.

\section{CONCLUSIONES}

Mientras se escribía este ensayo, se produjo el tercer derrocamiento de un presidente ecuatoriano en ocho años. Resulta imposible dejar de contraponer este tipo de hechos a la reflexión sobre el desarrollo de la ciencia política y preguntarse por la incidencia de la larga historia de inestabilidad política en la construcción de un espacio académico. Es innegable que aquellas incidencias, con su búsqueda permanente de mecanismos que faciliten la gobernabilidad y que mejoren las condiciones de la representación política, configuran un laboratorio ideal para desarrollar y mejorar explicaciones científicas de los fenómenos políticos. Sin embargo, al mismo tiempo, encierran a la reflexión en un horizonte restringido y fuertemente determinado por la necesidad de contar con respuestas urgentes a problemas inmediatos.

Esto explica, en gran medida, el bajo apego de los estudios políticos a las reflexiones de largo alcance, la escasa consideración de procesos de más amplio horizonte temporal (con las excepciones señaladas en el texto) e incluso el incipiente interés por el estudio de temas que demandan una visión relativamente alejada de las determinaciones coyunturales y la escasa acogida de perspectivas propiamente politológicas. Pero, esas condiciones no explican en su totalidad las dificultades existentes para constituir un campo específico de la disciplina. Es probable que algunas pistas se encuentren en el predominio de otras ciencias, la ausencia de formación especializada en las universidades, la herencia dejada por las perspectivas estructuralistas y el rechazo manifiesto a la delimitación de lo político como un campo de conocimiento autónomo.

Es evidente, en cualquier caso, que las condiciones para la investigación política en Ecuador han mejorado de manera sustancial con respecto a las décadas anteriores. Esto ha sido posible gracias la confluencia de varios factores, entre los cuales se destacan la reorientación de algunos académicos ecuatorianos hacia el campo de la ciencia política (con la consecuente adopción de perspectivas y métodos propios de esta disciplina), la creciente activa participación de académi- 
cos extranjeros interesados en el país, la formación de ecuatorianos en el exterior, y el desarrollo de bases de datos electorales y legislativas, así como una tendencia general a producir información sobre otros procesos políticos nacionales.

El futuro de la ciencia política ecuatoriana dependerá en buena medida en que las instituciones existentes puedan aprovechar estos factores para ofrecer una sólida formación teórica y metodológica a los alumnos de pregrado y postgrado, así como promover la producción, difusión y debate del conocimiento adquirido. Por un lado, es necesario mejorar la enseñanza de la Ciencia Política en el país, a través de la creación y fortalecimiento de programas politológicos en el pregrado y postgrado. Se debe impulsar -en la manera que sea posible- un pénsum común de estudios que privilegie la metodología de investigación, así como establezca un conjunto de obras claves para entender el desarrollo del pensamiento político en términos generales y específicamente en Ecuador. Por otro lado, es imprescindible promover y sustentar la investigación académica. En este sentido, es importante desarrollar redes de financiamiento entre universidades locales y extranjeras, agencias de cooperación y gobierno, para sustentar la investigación científica en Ecuador. De igual manera, es importante desarrollar espacios apropiados para debatir y publicar los hallazgos académicos en el país.

El reto de consolidar la ciencia política en Ecuador es sustantivo, pero impostergable en un país que está en constante espera de respuestas o explicaciones de su crónica inestabilidad. Ignorar la tarea pendiente equivaldría a condenar la producción de ciencia política a narrar diagnósticos políticos post mortem, en lugar de explotar su potencial de conocimiento científico para prevenir escenarios de crisis y contribuir a la toma de decisiones políticas con análisis rigurosos, oportunos y bien informados.

\section{REFERENCIAS}

Alcántara, Manuel. 1994. "Cuando hablamos de Ciencia Política, ¿de qué hablamos?". Revista Mexicana de Sociología 4: 147-178.

Almond, Gabriel. 2001. "Ciencia Política: la historia de la disciplina". En Nuevo Manual de Ciencia Política, editado por Robert Goodin y Hans-Dieter Klingemann. Madrid: Istmo.

Acosta, Alberto. 1996. "El bucaramismo en el poder". Nueva Sociedad 146 (junio-agosto): 6-15.

Acosta, Alberto et al. 1984. El Ecuador en las Urnas. Lucha Social y Lucha Política. Quito: El Conejo.

Andrade, Xavier. 2000. “Medios, imágenes y los significados políticos del "machismo"'. Ecuador Debate 49 (abril): 139-164. Andrade, Xavier. 2001. "Adiós cultura y hasta la vista cultura política. Sobre el tratamiento sociológico del regionalismo y populismo en Ecuador". Nueva Sociedad 175 (septiembre-octubre): 121-133.

Argones, Nelson. 1986. El juego del poder de Rodríguez Lara a Febres Cordero. Quito: CEN-INFOC.

Arias, Natalia. 1995. "Partidos Políticos: ¿Héroes o villanos?". Ecuador Debate 36 (diciembre): 49-65.

Ardaya, Gloria y Luis Verdesoto. 1996. "Ecuador de la expectativa por la nación a una sociedad sin expectativas". Nueva Sociedad 142 (marzo-abril): 16-21.

Ardaya, Gloria y Luis Verdesoto. 1999. "El sistema electoral ecuatoriano. Evaluación de las elecciones nacionales 1998, la crisis de 1999 y la agenda electoral pendiente". Cuadernos del Cendes 16 (41) (mayo-agosto): 83-112.

Ayala, Enrique. 1985. Lucha política y origen de los partidos políticos en Ecuador. Quito: Corporación Editora Nacional.

Barrera, Augusto. 2001. Acción colectiva y crisis política: el movimiento indígena ecuatoriano en la década de los noventa. La Rábida: Universidad Internacional de Andalucía.

Blankstein, George I. 1951. Ecuador: Constitutions and Caudillos. Berkeley: University of Los Angeles Press.

Burbano de Lara, Felipe. 1989. "Discurso populista, democracia y modernización". Ecuador Debate 17: 115-128.

Burbano de Lara, Felipe. 1998a. Cultura Política y democracia en el Ecuador: una aproximación a nuestros vacíos. Quito: CORDES. 
Burbano de Lara, Felipe (coord.). 1998b. El fantasma del populismo. Caracas: Nueva Sociedad.

Burbano de Lara, Felipe y Michel Rowland. 1998. Pugna de Poderes: Presidencialismo y Partidos en el Ecuador: 1979-1997.

Quito: CORDES-Fundación Konrad Adenauer y Agencia de Cooperación Española.

Bustamante, Fernando.1984. "La ideología de los partidos políticos ecuatorianos". En El Ecuador en las Urnas. Lucha Social y Lucha Política, editado por Alberto Acosta et al. Quito: El Conejo.

Bustamante, Fernando. 1997. "La cultura política y ciudadana en el Ecuador". En Ecuador: un problema de gobernabilidad, editado por varios autores. Quito: CORDES-PNUD.

Bustamante, Fernando. 2000. "Los partidos como orientaciones culturales". Íconos 9 (abril): 88-97.

Buttolph J ohnson, J anet, Richard A. J oslyn y H. T. Reynolds. 2001. Political Science Research Methods. Washington: Congressional Quarterly Press.

Conaghan, Catherine M. 1988. Restructuring domination: industrialists and the state in Ecuador, Pitt Latin American series. Pittsburgh, Pa.: University of Pittsburgh Press.

Conaghan, Catherine. 1995. "Politicians against Parties: Discord and Disconneection in Ecuador's Party System". En Building Democratic Institutions, editado por Scott Mainwaring y Timothy Scully. Stanford: Stanford University Press.

CORDES. 1996. Ecuador: Un problema de gobernabilidad. Ecuador: CORDES.

CORDES. 1999. La ruta de la gobernabilidad. Quito: CORDES y Cooperación Española.

Córdoba, Gabriela. 2003. "Derrota y triunfo del gran elector: elecciones 2002 en Ecuador". Íconos 16 (mayo): 11-16.

Corkil, David y David Cubit. 1992. "Capitalist, Technocrats, and politicians: Economic policy making and Democracy in the Central Andes". En Issues in Democratic Consolidation: The New South American Democracies in Comparative Perspective, editado por Scott Mainwaring, Guillermo O'Donnell y Samuel Valenzuela. Notre Dame: University of Notre Dame Press.

Cueva, Agustín. 1988. El proceso de dominación política en el Ecuador. Quito: Sudamericana.

De la Torre, Carlos. 1997. "Los usos políticos de las categorías pueblo y democracia”. Ecuador Debate 41 (agosto): 62-77. De la Torre, Carlos. 2000. La seducción Velasquista. Quito: Ediciones Libri Mundi y FLACSO.

De la Torre, Carlos. 2001. "Política y economía en los nuevos y viejo populismos". Ecuador Debate 53 (agosto): 73-86.

Echeverría, Julio. 1994. "La construcción social de la política: notas sobre la crisis del sistema de partidos en el Ecuador". Nueva Sociedad 134 (noviembre- diciembre): 130-141.

Echeverría, Julio. 1997. La democracia bloqueada. Quito: Letras.

Egas, J osé María. 1983. "Contradicciones y relación de fuerzas en el proceso electoral”. Ecuador Debate 4 (diciembre): 7-21. Fitch, Samuel. 1977. The Military Coup D'Etat as a Political Process: Ecuador, 1948-1966. Baltimore: J ohns Hopkins University Press.

Freidenberg, Flavia. 2001. "Ecuador". En Partidos Políticos de América Latina, editado por Manuel Alcántara Sáez y Flavia Freidenberg. Salamanca: Ediciones Universidad de Salamanca.

Freidenberg, Flavia. 2003. Jama, Caleta y Camello. Las estrategias de Abdalá Bucaram y el PRE para ganar elecciones. Quito: Corporación Editora Nacional.

Freidenberg, Flavia. 2004 "Fracturas sociales, competencia política y sistemas de partidos en Ecuador: la traducción política de un cleavage étnico". En Etnicidad, Autonomía y Gobernabilidad en América Latina, editado por Salvador Martí i Puig. Salamanca: Ediciones Universidad de Salamanca, 101-138.

Freidenberg, Flavia. 2005. "Izquierda vs. Derecha. Polarización ideológica y competencia política en el sistema de partidos ecuatoriano". Ponencia presentada en el Encuentro de Latinoamericanistas, realizado en Tordesillas entre el 26 y el 28 de mayo.

Freidenberg, Flavia y Manuel Alcántara Sáez. 2001. Los dueños del poder: Partidos políticos en Ecuador (1978-2000). Quito: FLACSO- Sede Ecuador.

Goodin, Robert y Hans-Dieter Klingemann. 2001. Nuevo Manual de Ciencia Política. Madrid: Istmo.

Guerrero, Andrés. 1998. "Ciudadanía, frontera étnica y compulsión binaria". Íconos 4 (noviembre-diciembre): 106-118.

Guerrero, Rafael. 1990. "La crisis de Guayaquil y los nuevos populismos". Ecuador Debate 21: 16-23.

Guerrero, Rafael. 1994. Regionalismo y democracia social en los orígenes del 'CFP'. Quito: Centro Andino de Acción Popular Hurtado, Osvaldo. 1977. El poder político en el Ecuador. Quito: Planeta.

Ibarra, Hernán. 1994. “Tendencias sociales y políticas en las elecciones de mayo de 1994”. Ecuador Debate 32 (agosto): 90-100. 
Icaza, Patricio. 1983. Apuntes para la historia del movimiento obrero. Quito: Universidad Central.

Isaacs, Anita. 1990. "Ecuador: Democracy standig the test of time?". En Constructing Democratic Governance. South America in the 1990s, editado por J orge Domínguez y Abraham Lowenthal. Baltimore y Londres: J ohns Hopkins, 42-57.

Landau, David. 2001. Policy or Pork? Presidential Strategy Choice and the Legislature in Ecuador. Cambridge: Department of Government, Harvard College.

León, Jorge. 1987. Elecciones, votos y partidos. Quito: CEDIME.

León, J orge. 1994. "El fin de un ciclo político electoral: el regreso de las elites tradicionales, apatía y cambio". Ecuador Debate 32 (agosto): 72-89.

León Velasco, Juan. 1992. Elecciones en el Ecuador: Concejales Cantonales 1978-1990. Quito: CIESA.

Maiguaschca, Juan. 1994. Historia y región en el Ecuador: 1830-1930. Quito: Corporación Editora Nacional.

Massal, J ullie y Marcelo Bonilla. 2000. Los movimientos sociales en las democracias andinas. Quito: FLACSO.

Martz, John.1972. Conflicting political culture and the quest for progress. Boston: Allyn and Bacon.

Martz, John. 1983. "Populist Leadership and the Party Caudillo: Ecuador and the CFP, 1962-81". Studies in Comparative International Development 18 (3) (fall): 22-49.

Mateos Díaz, Araceli y Manuel Alcántara. 1998. "Los diputados ecuatorianos: actitudes, valores y percepciones políticas". Quito: Programa de Apoyo al Sistema de Gobernabilidad Democrática-CORDES, FLACSO, Universidad Andina Simón Bolívar y Maestría en Ciencia Política y Administración Pública, Pontificia Universidad Católica del Ecuador.

Mejía Acosta, Andrés. 1998. Partidos Políticos: El eslabón perdido de la representación. Documento de Trabajo 5. Quito: Fundación Konrad Adenauer Stiftung y CORDES.

Mejía Acosta, Andrés. 1999. "Indiciplina y Lealtad en el Congreso". Iconos 6 (enero):13-21.

Mejía Acosta, Andrés. 2000. "Y sin embargo... se mueve: Patrones de coherencia y certidumbre en el Congreso Nacional". Iconos 9 (abril): 64-78.

Mejía Acosta, Andrés. 2002. Gobernabilidad Democrática. Quito: Fundación Konrad Adenauer.

Mejía Acosta, Andrés. 2003. "La reelección legislativa en Ecuador: conexión electoral, carreras legislativas y partidos políticos (1979-2003)". En El legislador a examen. El debate sobre la reelección legislativa en México: una perspectiva histórica e institucional, editado por F. F. Dworak. México: Fondo de Cultura Económica.

Mejía Acosta, Andrés. 2004. Ghost Coalitions: Economic Reforms, Fragmented Legislatures and Informal Institutions in Ecuador. Notre Dame: Department of Political Science, University of Notre Dame,.

Mejía Acosta, Andrés. 2006. "Crafting Legislative Ghost Coalitions in Ecuador: Informal Institutions and Economic Reform in an Unlikely Case". En Informal Institutions and Democracy: Lessons from Latin America, editado por G. Helmke y S. Levistky. J ohns Hopkins University Press.

Mejía Acosta, Andrés, Caridad Araujo, Aníbal Pérez Liñán, Sebastián M. Saiegh y Simón Pachano. 2004. Political Institutions, Policymaking Processes, and Policy Outcomes in Ecuador. FLACSO-InterAmerican Development Bank.

Menéndez Carrión, Amparo. 1986. La conquista del voto: de Velasco a Roldós. Quito: Corporación Editora Nacional. Menéndez Carrión, Amparo. 1991. Ecuador, la democracia esquiva. Quito: Instituto Latinoamericano de Investigación.

Mijeski, Kenneth y Scott Beck. 1998. Mainstreaming the indigenous movement in Ecuador: The electoral strategy. Paper presentado en el XXI Congreso Internacional de la Asociación de Estudios Latinoamericanos, Chicago, Illionois. 24-26 de septiembre.

Mills,Nick D. 1984. Crisis, Conflicto y Consenso. Ecuador: 1979-1984. Quito: Corporación Editora Nacional.

Montúfar, César. 1990. "Ecuador: elecciones presidenciales de 1988". Revista Mexicana de Sociología Año LII/ 4 (octubrediciembre): 155-176.

Montúfar, César. 2000. La reconstrucción neoliberal. Febres Cordero o la estatización del neoliberalismo en el Ecuador (1984-1988). Quito: Ediciones Abya Yala.

Naranjo, Alexis. 1993. "Las cámaras de la producción y la política". Ecuador Debate 30 (diciembre): 155-168.

Ojeda, Lautaro. 1971. Mecanismos y articulaciones del caudillismo velasquista. Quito: J NP.

Ojeda, Lautaro. 1983. "Clientelismo y Microoligarquía en la Cuenca del Guayas". Ecuador Debate 3 (agosto): 106-114.

Quintero, Rafael. 1980. El mito del populismo. Quito: FLACSO. 
Quintero, Rafael. 2002. Entre el hastío y la participación ciudadana. Partidos y elecciones en Ecuador (2000-2002). Quito: Abya Yala.

Pachano, Simón. 1991. Los diputados, una elite política. Quito: Corporación Editora Nacional.

Pachano, Simón. 1996. Democracia sin sociedad. Quito: Corporación Editora Nacional.

Pachano, Simón. 1997. “Ecuador. Desorden populista y caos institucional”. Nueva Sociedad 157 (septiembre-octubre): 4-9.

Pachano, Simón. 1998. La representación caótica. Quito: FLACSO.

Pachano, Simón. 1999. "Problemas de representación y partidos políticos en Ecuador". En Partidos políticos y representación en América Latina, coordinado por Thomas Manz y Moura Zuazo. Caracas: ILDIS-Edit. Nueva Sociedad - Friederich Ebert.

Pachano, Simón. 2004. "El Territorio de los Partidos". En Partidos políticos en la región andina: entre la crisis y el cambio, editado por Simón Pachano. Lima: Ágora Democrática-IDEA.

Sánchez, Francisco. 1999. "Introducción al sistema electoral ecuatoriano". Documentos de Trabajo 3. Quito: FEUCE y Maestría en Ciencias Políticas y de la Administración Pública (PUCE).

Sánchez, Francisco y Flavia Freidenberg. 1998. "El proceso de incorporación política de los sectores indígenas en el Ecuador. Pachakutik, un caso de estudio". América Latina Hoy 19 (julio): 65-79.

Sánchez Parga, José. 1998. La Pugna de Poderes. Quito: Abya-Ayala Ediciones.

Seligson, Mitchell y A. Polibio Córdova C. 2002. Auditoría de la Democracia. Ecuador. Quito: Proyecto de Opinión Pública Latinoamericana de la Universidad de Pittsburgh y CEDATOS.

Van Cott, Donna.1999. Indigenous Peoples and Democracy in Latin America. New York: St. Martin's Press.

Yashar, Deborah. 2005. Contesting Citizenship:Indigenous Movements and Democracy in Latin America. Cambridge: Cambridge University Press.

Andrés Mejía Acosta es Doctor en Ciencia Política por la Universidad de Notre Dame (2004), Estados Unidos, y actualmente es investigador del Departamento de Ciencia Política en la Universidad de British Columbia, Canadá. Su última publicación es Ghost Coalitions: Economic Reforms, Fragmented Legislatures and Informal Institutions in Ecuador (Ph.D. Dissertation, Department of Political Science, Notre Dame, University of Notre Dame, 2004).

(E-mail: andres.mejia@ubc.ca)

Flavia Freidenberg es Doctora en Ciencia Política por la Universidad de Salamanca y profesora del Instituto Iberoamericano de Estudios de Iberoamérica y Portugal. Su más reciente libro tiene por título Jama, Caleta y Camello: Las Estrategias de Abdalá Bucaram y el PRE para ganar las elecciones (2003, Quito, Ecuador: Corporación Editora Nacional). (E-mail: flavia@usal.es)

Simón Pachano es Candidato a Doctor en Ciencia Política por la Universidad de Salamanca y profesor de la Facultad Latinoamericana de Ciencias Sociales (FLACSO-Ecuador). Su último libro es Partidos Políticos en la Región Andina: entre la crisis y el cambio (2004, Lima, Perú: Ágora Democrática).

(E-mail: spachano@flacso.org.ec) 